\title{
Enfermedades invasoras por Streptococcus pyogenes 2005-2013. Hospital Pediátrico del Centro Hospitalario Pereira Rossell, Uruguay
}

\author{
Alejandra Vomero, Gabriela García, Soledad Pandolfo, Carlos Zunino, \\ María Ambrosoni, Gabriela Algorta y M. Catalina Pírez
}

Invasive Streptococcus pyogenes diseases 2005-2013. Pediatric Hospital Pereira Rossell Uruguay

Introduction: Streptococcus pyogenes infection causes a wide spectrum of clinical manifestations. Invasive disease (ID) is defined by the isolation of the microorganism from sterile sites. Objective: To analyze the clinical, epidemiological and molecular characteristics of ID by S.pyogenes in children hospitalized at Pediatric Hospital Pereira Rossell, from January 2005 to January 2013. Materials and Methods: A descriptive retrospective study was done in cases with isolation of S.pyogenes from sterile sites. Epidemiological variables, disease characteristics, laboratory parameters, treatment regimen received, hospitalization days and clinical outcome were analyzed. The presence of 4 genes encoding for virulence factors and chromosome profile studied by pulsed-field electrophoresis were done in the isolated strains. Results: A total of 42 cases (rate: 4.6 out of 10,000 admissions) were detected, from which 32 were analyzed. Average age was 44.7 months ( $14 / 32<2$ years of age). In 5 cases, the portal of entry was identified. Clinical presentations were: osteoarticular infections $(n=15)$, shock $(n=6)$, skin and softtissue infections $(n=5)$, pneumonia $(n=3)$ and bacteremias $(n=3)$. Twenty cases required surgical procedures and 13 required intensive care admission. Average hospital stay was 17 days and one patient died. Molecular studies were performed in five strains; 4 different toxin profiles and pulsotypes were identified. Discussion: The incidence of ID at our hospital is similar to other series in the region. A better knowledge of clinical presentation and its relation with molecular characteristics represents a challenge.

Key words: Streptococcus pyogenes, invasive disease, emm type.

Palabras clave: Streptococcus pyogenes, enfermedades invasoras, tipo emm.

\section{Introducción}

S treptococcus pyogenes es una cocácea grampositiva que produce un amplio espectro de patologías desde leves como faringitis hasta presentaciones clínicas muy graves que pueden provocar la muerte. Las enfermedades invasoras (EI) son definidas por el aislamiento del microorganismo en sitios normalmente estériles ${ }^{1}$. En este grupo de EI se incluyen tres síndromes que pueden superponerse clínicamente: síndrome de shock tóxico (SST), fascitis necrosante, y enfermedades invasoras no asociadas a las anteriores: bacteriemia, neumonía, osteomielitis, celulitis necrosante y artritis séptica. Las EI requieren un diagnóstico y tratamiento precoz ya que producen alta morbi-mortalidad ${ }^{2}$.

Streptococcus pyogenes posee diversos factores de virulencia, entre ellos su cápsula de ácido hialúronico, el factor de opacidad del suero, la proteína $\mathrm{F}$, la peptidasa $\mathrm{C} 5 \mathrm{a}$, las hemolisinas O y S, SpeB (exotoxina B pirógena estreptocóccica, potente proteasa), las exotoxinas pirogénicas estreptocóccicas: SpeA y SpeC, el superantígeno estreptocóccico (SSA) y la proteína M. Esta última constituye el principal factor de virulencia, está codificada por el gen emm y mediante la secuenciación de extremo 5 ' hipervariable permite una subdivisión en más de 200 tipos $\mathrm{emm}^{3}$. A partir de la década del 80 ' se produjo una re-emergencia de enfermedades invasoras por este patógeno, aunque no de la misma magnitud que la observada en la era pre-antibiótica. Nuevas manifestaciones clínicas, no descritas anteriormente, como el SST y la fascitis necrosante de extrema gravedad y evolución hiperaguda, emergieron como causa de muerte en pacientes sin patologías de base ni factores de riesgo definidos ${ }^{4}$. Esta re-emergencia puede estar relacionada a cambios en la distribución o aparición de determinados tipos emm o de las exotoxinas pirogénicas 5 .

En Uruguay, las infecciones causadas por S. pyogenes no son de denuncia obligatoria, por lo que se desconoce la carga de enfermedad atribuible a ellas. Existen escasas comunicaciones nacionales sobre el tema. Picón y cols. ${ }^{6}$, en 1994 publicaron dos casos de infecciones pulmonares graves por S. pyogenes y Prego y cols., en los años 1997
Universidad de la República. Montevideo, Uruguay. Facultad de Medicina, Departamento de Pediatría. Hospital Pediátrico. Centro Hospitalario Pereira Rossell (HP-CHPR) - ASSE. Montevideo, Uruguay.

Laboratorio de Microbiología,

Laboratorio Central HP-CHPR- ASSE. Departamento de Laboratorios del Ministerio de Salud Pública (DLSP)

Recibido: 26 de febrero de 2014 Aceptado: 28 de julio de 2014

Correspondencia a: Carlos Zunino careduzu@hotmail.com 
y 2001 reportaron una serie de casos de complicaciones graves por este microorganismo en niños previamente sanos cursando varicela ${ }^{7,8}$.

El interés de este estudio es profundizar el conocimiento de la epidemiología de las EI por $S$. pyogenes y su presentación clínica en niños, a la vez que comunicar los avances que se han realizado en la caracterización de los factores de virulencia de las cepas aisladas en Uruguay, utilizando técnicas de biología molecular.

En los últimos años, en el Departamento de Laboratorios del Ministerio de Salud Pública (DLSP) se han implementado técnicas que permiten investigar diferentes factores de virulencia en las cepas referidas a dicha institución.

\section{Objetivo}

Analizar las características clínicas y epidemiológicas de los niños hospitalizados con EI por $S$. pyogenes en el Hospital Pediátrico del Centro Hospitalario Pereira Rossell (HP-CHPR) desde el 1 de enero de 2005 al 31 de enero de 2013. Describir los factores de virulencia encontrados en las cepas estudiadas.

\section{Materiales y Métodos}

Estudio descriptivo y retrospectivo. Se buscó en la base de datos del Laboratorio de Microbiología del Laboratorio Central del HP-CHPR todos los casos en los que se aisló $S$. pyogenes de sitios normalmente estériles durante el período de estudio. De los 42 casos encontrados se pudo analizar todas las variables en 32, ya que los registros clínicos de los 10 restantes eran inaccesibles. Los cultivos y la identificación del agente aislado se realizaron por técnicas microbiológicas habituales ${ }^{9}$. De las historias clínicas se indagaron las siguientes variables: edad, sexo, factores predisponentes (pérdida de continuidad cutánea, traumatismo, foco de infección a distancia, varicela, inmunodepresión, contacto epidemiológico y consumo previo de antimicrobianos, sitio de aislamiento del agente causal, recuento de leucocitos, proteína $\mathrm{C}$ reactiva $(\mathrm{PCR})$, tratamientos médico y quirúrgico, evolución, días de hospitalización y estadía en Unidad de Tratamiento Intensivo (UTI). Se consideró patológico un recuento de leucocitos $<$ a $5.000 / \mathrm{mm}^{3}$ o > a $15.000 / \mathrm{mm}^{3}$ y $\geq$ PCR $20 \mathrm{mg} / \mathrm{L}$.

\section{Definiciones de casos}

Enfermedad invasora: Se definió como aquel caso en el que se aisló $S$. pyogenes de sitios normalmente estériles (sangre, líquido pleural, líquido sinovial, tejidos blandos y hueso).

Síndrome de shock tóxico: Se consideró como tal, el caso de EI que tuvo rápida evolución a shock y falla multiorgánica ${ }^{2,10}$.
Fascitis necrosante: Un caso de infección de tejidos subcutáneos y fascias, que presentó necrosis extensa y rápida diseminación ${ }^{2}$.

Bacteriemia oculta se definió por el aislamiento de $S$. pyogenes en sangre, en un niño febril sin aspecto clínico de toxicidad ni foco clínico evidente ${ }^{11}$.

Para la definición de celulitis, neumonía, osteomielitis y/o artritis se utilizaron los criterios diagnósticos publicados en las Pautas Nacionales de Atención Pediátrica ${ }^{12}$.

Se consideró infección intrahospitalaria aquel caso en que la EI por S. pyogenes no estuvo presente clínicamente, ni en el período de incubación cuando el niño ingresa al hospital, considerando $48 \mathrm{~h}$ previas al ingreso como período de incubación.

De las cepas de $S$. pyogenes aisladas en el laboratorio de los niños incluidos en esta serie, cinco habían sido referidas a continuación al DLSP donde se realizó la caracterización molecular. Se tipificó por secuenciación el gen $\mathrm{emm}^{3}$ y se obtuvieron perfiles cromosómicos por digestión del ADN con la enzima de restricción SmaI, resueltos por electroforesis en gel de campos pulsados (en inglés PFGE). De acuerdo a la inspección visual y siguiendo los criterios de Tenover ${ }^{13}$, se identificaron los pulsotipos obtenidos. Además, se estudió la presencia/ausencia de genes que codifican para factores de virulencia: SpeA, SpeB, SpeC y SSA ${ }^{14}$. Los resultados se compararon con la base de datos existente en el DLSP ${ }^{15}$.

Este estudio fue autorizado por la Dirección del HP CHPR y aprobado por su Comité de Ética. Por su carácter retrospectivo y protección de la confidencialidad de los datos que identifican al paciente se otorgó una dispensa al consentimiento informado.

\section{Resultados}

Durante el período se hospitalizaron 42 niños con EI causada por $S$. pyogenes (4,6 por 10.000 ingresos). Se analizaron todas las variables en 32 . La mediana de edad fue 44,7 meses (1-156 meses), 14 tenían menos de 2 años de edad. Eran varones 22. En la Tabla 1 se muestra la distribución según la presentación clínica. No tenían comorbilidad 25; de los que la presentaron, en uno se realizó el diagnóstico de infección por VIH en la internación, cuatro eran asmáticos, un niño tenía dermatitis atópica y otro una fístula perianal congénita.

Un caso se clasificó como infección intrahospitalaria.

Los sitios anatómicos/fluidos de aislamiento del microorganismo se detallan en la Tabla 2; en su mayoría $(62,5 \%)$ fue la sangre.

\section{Presentaciones clínicas}

En relación a las formas de presentación clínica: en 15 niños el diagnóstico fue infección osteo-articular, seis 
Tabla 1. Enfermedades invasoras por Streptococcus pyogenes. Hospital Pediátrico del Centro Hospitalario Pereira Rossell 1-1-2005 al 31-1-2013. Características bio-demográficas según presentación clínica

\begin{tabular}{|c|c|c|c|c|c|c|}
\hline & $\begin{array}{c}\text { Grupo } 1 \\
\text { Infecciones } \\
\text { osteo-articulares }\end{array}$ & $\begin{array}{c}\text { Grupo 2 } \\
\text { Infecciones } \\
\text { de tejidos blandos }\end{array}$ & $\begin{array}{c}\text { Grupo3 } \\
\text { Infecciones } \\
\text { pleuro-pulmonares }\end{array}$ & $\begin{array}{l}\text { Grupo } 4 \\
\text { SST }\end{array}$ & $\begin{array}{c}\text { Grupo } 5 \\
\text { Bacteriemia oculta }\end{array}$ & Total \\
\hline $\begin{array}{l}\text { Edad (meses) } \\
\text { Media (rango) }\end{array}$ & $\begin{array}{c}67,3 \\
(14-156)\end{array}$ & $\begin{array}{c}34 \\
(6-120)\end{array}$ & $\begin{array}{l}20,6 \\
(2-36)\end{array}$ & $\begin{array}{c}31,5 \\
(2-120)\end{array}$ & $\begin{array}{c}3,3 \\
(1-8)\end{array}$ & $\begin{array}{l}\bar{x} 44,7 \\
(1-156)\end{array}$ \\
\hline Sexo (M/F) & $10 / 5$ & $5 / 0$ & $3 / 0$ & $3 / 3$ & $1 / 2$ & $22 / 10$ \\
\hline Total n (\%) & $15(46,8)$ & $5(15,6)$ & $3(9,3)$ & $6(18,7)$ & $3(9,3)$ & $32(100)$ \\
\hline
\end{tabular}

casos se presentaron como SST, cinco como infección de tejidos blandos (dos fascitis necrosante, tres celulitis necrosante), tres infecciones pleuro-pulmonares y otros tres correspondieron a bacteriemia oculta.

\section{Factores predisponentes y manifestaciones clínicas}

Los factores predisponentes identificados fueron: impétigo en cuatro niños, traumatismo de leve intensidad en siete y antecedente de pérdida de solución de continuidad en dos casos (una cirugía reparadora de un defecto congénito y una herida de piel suturada). El uso de antimicrobianos previo al inicio de la enfermedad se reportó en cuatro casos (Tabla 3). No se registraron niños con varicela como factor predisponente.

Todos los niños tuvieron fiebre desde el inicio de la enfermedad. El tiempo de evolución previo al ingreso fue de 4 días (1-30 días).

El recuento medio de leucocitos fue $17,8 \times 10^{3}$ céls/ $\mathrm{mm}^{3}\left(6,5-37,5 \times 10^{3}\right.$ céls $\left./ \mathrm{mm}^{3}\right)$. La media del valor de proteína $\mathrm{C}$ reactiva (PCR) fue: $345 \mathrm{mg} / \mathrm{L}$.

Todos recibieron antimicrobianos intravenosos, el tratamiento empírico inicial fue adecuado para S. pyogenes. Requirieron procedimiento quirúrgico 17 niños. La media de hospitalización fue de 17,1 días, con un rango de 2 a 43 días. La media de estadía en UTI fue de 3,7 días, con un rango de 0 a 26 (Tabla 4).
Tabla 2. Enfermedades invasoras por Streptococcus pyogenes.

Hospital Pediátrico del Centro Hospitalario Pereira Rossell 1-1-2005 al 31-1-2013. Lugar de aislamiento microbiano

\begin{tabular}{lcc|} 
Sitios normalmente estériles & $\mathbf{n}$ & Frecuencia \% \\
Sangre & 14 & 43,8 \\
Líquido articular & 10 & 31,3 \\
Líquido pleural & 2 & 6,2 \\
Sangre y líquido pleural & 2 & 6,2 \\
Sangre y hueso & 3 & 9,4 \\
Sangre y material de drenaje quirúrgico & 1 & 3,1 \\
Total & 32 & 100 \\
\hline
\end{tabular}

\section{Caso fatal}

Se registró un fallecimiento, se trataba de una lactante con tres meses de vida, portadora de una fistula perineal congénita, que ingresó al hospital para cirugía de coordinación de anorectoplastía. En el block quirúrgico se le instaló una vía venosa central subclavia derecha. En el post-operatorio inmediato permaneció en la UTI. Evolucionó inicialmente de forma favorable, sin complicaciones. Al quinto día del post-operatorio aparecieron un aspecto séptico, un hematoma a nivel de la vena subclavia

Tabla 3. Enfermedades invasoras por Streptococcus pyogenes. Hospital Pediátrico del Centro Hospitalario Pereira Rossell 1-1-2005 al 31-1-2013. Factores predisponentes según presentación clínica

\begin{tabular}{|c|c|c|c|c|c|c|}
\hline Factores predisponentes & $\begin{array}{c}\text { Grupo } 1 \\
\text { Infecciones osteo- } \\
\text { articulares }\end{array}$ & $\begin{array}{c}\text { Grupo } 2 \\
\text { Infecciones } \\
\text { de tejidos blandos }\end{array}$ & $\begin{array}{c}\text { Grupo3 } \\
\text { Infecciones pleuro } \\
\text { pulmonares }\end{array}$ & $\begin{array}{c}\text { Grupo } 4 \\
\text { SST }\end{array}$ & $\begin{array}{c}\text { Grupo } 5 \\
\text { Bacteriemia oculta }\end{array}$ & Total \\
\hline Lesión de piel (impétigo) & 1 & 3 & 0 & 0 & 0 & 4 \\
\hline Trauma & 5 & 0 & 0 & 1 & 0 & 6 \\
\hline Trauma y procedimiento quirúrgico & 1 & 0 & 0 & 0 & 0 & 1 \\
\hline Procedimiento quirúrgico & 0 & 0 & 0 & 1 & 0 & 1 \\
\hline Antimicrobianos previos & 2 & 1 & 0 & 0 & 1 & 4 \\
\hline Total & 9 & 4 & 0 & 2 & 1 & 16 \\
\hline
\end{tabular}


Tabla 4. Enfermedades invasoras por Streptococcus pyogenes. Hospital Pediátrico del Centro Hospitalario Pereira Rossell 1-1-2005 al 31-1-2013. Parámetros de laboratorio, tratamiento y media de internación según presentación clínica

\begin{tabular}{|c|c|c|c|c|c|c|}
\hline & $\begin{array}{c}\text { Grupo } 1 \\
\text { Infecciones } \\
\text { osteo-articulares }\end{array}$ & $\begin{array}{c}\text { Grupo } 2 \\
\text { Infecciones } \\
\text { de tejidos blandos }\end{array}$ & $\begin{array}{c}\text { Grupo3 } \\
\text { Infecciones } \\
\text { pleuro-pulmonares }\end{array}$ & $\begin{array}{c}\text { Grupo } 4 \\
\text { SST }\end{array}$ & $\begin{array}{c}\text { Grupo } 5 \\
\text { Bacteriemia oculta }\end{array}$ & Total \\
\hline $\begin{array}{l}\text { Leucocitos }\left(\times 10^{3} \mathrm{~mm}^{3}\right) \\
\text { Media (rango) }\end{array}$ & $\begin{array}{c}18,3 \\
(7,4-37,5)\end{array}$ & $\begin{array}{c}15,5 \\
(9,1-22,6)\end{array}$ & $\begin{array}{c}18,7 \\
(6,5-33)\end{array}$ & $\begin{array}{c}16,5 \\
(6,6-23)\end{array}$ & $\begin{array}{c}20,2 \\
(17,6-24,3)\end{array}$ & $\begin{array}{c}17,8 \\
(6,5-37,5)\end{array}$ \\
\hline $\begin{array}{l}\text { Proteína C reactiva (mg/L) } \\
\text { Media (rango) }\end{array}$ & $\begin{array}{c}91,9 \\
(0-242)\end{array}$ & $\begin{array}{c}97,6 \\
(53-150)\end{array}$ & $\begin{array}{c}173,6 \\
(176-345)\end{array}$ & $\begin{array}{c}113,0 \\
(77,8-160)\end{array}$ & $\begin{array}{c}75,0 \\
(24-126)\end{array}$ & $\begin{array}{c}107 \\
(0-345)\end{array}$ \\
\hline Tratamiento quirúrgico n (\%) & 8 & 3 & 3 & 3 & 0 & 17 \\
\hline $\begin{array}{l}\text { Días de hospitalización } \\
\text { Media (rango) }\end{array}$ & $\begin{array}{c}17,8 \\
(5-43)\end{array}$ & $\begin{array}{c}21,4 \\
(2-40)\end{array}$ & $\begin{array}{c}20,3 \\
(19-22)\end{array}$ & $\begin{array}{c}15,6 \\
(4-28)\end{array}$ & $\begin{array}{c}5,6 \\
(4-9)\end{array}$ & $\begin{array}{c}17,1 \\
(2-43)\end{array}$ \\
\hline $\begin{array}{l}\text { Días en UTI } \\
\text { Media (rango) }\end{array}$ & $\begin{array}{c}1,7 \\
(6-26)\end{array}$ & $\begin{array}{c}5,8 \\
(0-13)\end{array}$ & $\begin{array}{c}6,3 \\
(0-14)\end{array}$ & $\begin{array}{c}7,6 \\
(4-19)\end{array}$ & 0 & $3,7(0-26)$ \\
\hline
\end{tabular}

Tabla 5. Enfermedades invasoras por Streptococcus pyogenes. Hospital Pediátrico del Centro Hospitalario Pereira Rossell 1-1-2005 al 31-1-2013. Perfiles de toxinas

\begin{tabular}{llllll} 
Tipo de emm & speB & speA & speC & ssa & Presentación clínica \\
emm1 & pos & pos & neg & neg & SST, infección osteo-articular \\
emm12 & pos & neg & pos & neg & Infección pleuro-pulmonar \\
emm3 & pos & pos & neg & pos & SST \\
emm4 & pos & neg & pos & pos & SST \\
\hline
\end{tabular}

Los pacientes que presentaron SST e infección pleuro-pulmonar requirieron ingreso a UTI.

Figura 1. Enfermedades invasoras por Streptococcus pyogenes. Hospital Pediátrico del Centro Hospitalario Pereira Rossell 1-1-2005 al 31-1-2013. Caracterización de las cepas $S$. pyogenes. ADN cromosómico digerido con Smal, resuelto por PFGE. Carril 1 y 7: R6, cepa de referencia, carril 2 y 3 : cepas emm1, carril 4: cepa emm12, 5: cepa emm3 y carril 6: cepa emm4.

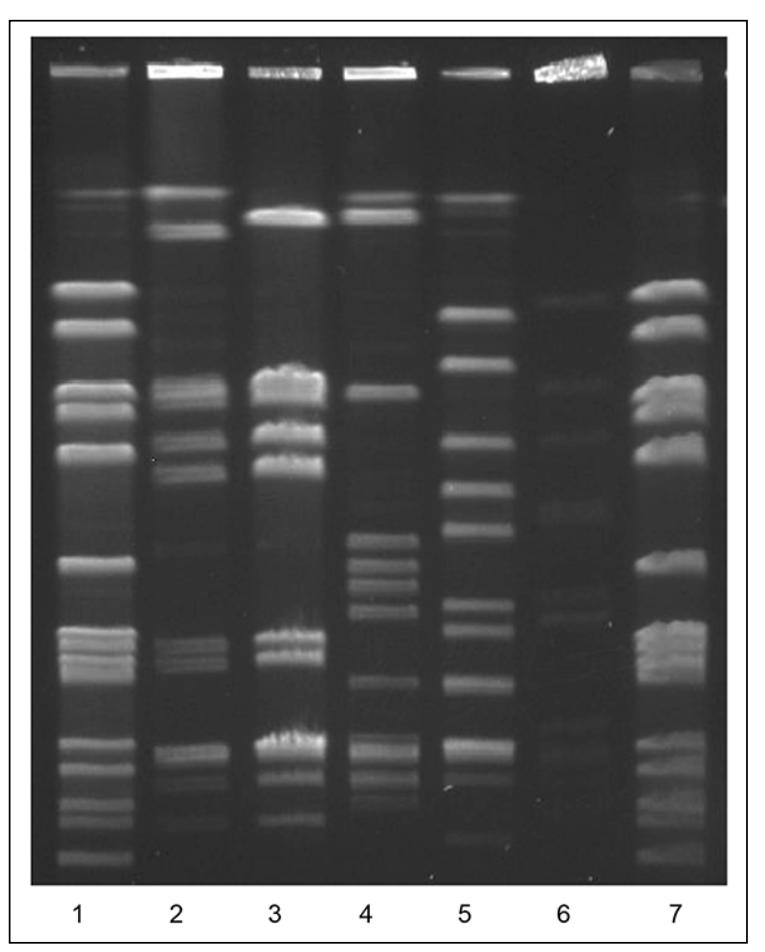

derecha y una efusión pleural derecha. Se colocó drenaje de tórax, obteniéndose un líquido hemo-purulento fétido. Evolucionó con shock refractario a volumen e inotrópicos, falleciendo a las $8 \mathrm{~h}$. En la necropsia se describieron: hepato-esplenomegalia, empiema y signos de sepsis. Tanto en el hemocultivo como en el cultivo de líquido pleural se desarrolló $S$. pyogenes. Se consideró este caso como infección intrahospitalaria.

\section{Estudio de biología molecular}

Se caracterizó molecularmente las cinco cepas enviadas al DLSP. Mediante secuenciación del gen emm se obtuvieron cuatro tipos: emm 1 (2 cepas), emm3, emm4 y emm 12. Por reacción de polimerasa en cadena (RPC) se identificaron cuatro perfiles de toxinas (Tabla 5) y cuatro pulsotipos por PFGE (Figura 1). Las cepas con tipo emm 1 compartían el mismo perfil de toxinas y los pulsotipos se diferenciaban en tres bandas; uno de ellos se consideró un subtipo del otro. Las cepas fueron aisladas de un niño con infección osteo-articular y en otro con SST. Las cepas tipo emm3 y 4 se aislaron en niños que presentaron SST. El tipo emm 12 se aisló en un niño con empiema pleural.

Solamente las cepas con tipo emm 1 y 3 (n: 3) tenían presente el gen de la exotoxina SpeA.

\section{Discusión}

Este es el primer estudio en Uruguay que permite aproximarse a la incidencia de hospitalización de niños con EI por $S$. pyogenes. La tasa de admisiones debidas a EI por este microorganismo coincide con la encontrada en otros estudios realizados en la región ${ }^{16,17}$. En esta serie, la edad media fue de 44,7 meses y en su mayoría eran niños previamente sanos, coincidiendo con las características clínicas descritas en la literatura médica ${ }^{1,16,18,19}$. 
En 16 pacientes se encontraron factores predisponentes, eran infecciones o soluciones de continuidad de la piel o traumatismos. En ninguno se registró varicela. La infección por el virus varicela-zoster es uno de los factores predisponentes más frecuente en niños comunicado en la literatura médica ${ }^{16}$. En las series anteriores de casos nacionales y regionales ningún niño había recibido la vacuna anti-varicela ${ }^{6,7,16,17}$. En Uruguay se comenzó la vacunación universal contra esta enfermedad en el año $1999^{20}$. El no encontrar casos asociados a esta infección puede ser el primer dato del impacto de esta estrategia en la prevención de las EI por $S$. pyogenes. En esta serie, la mayoría de los niños bajo un año de edad carecían de factores predisponentes; la corta edad en sí misma constituye un factor de riesgo para la adquisición de enfermedades invasoras ${ }^{21}$.

La fiebre es referida como un síntoma inicial constante, lo que se corroboró en estos pacientes. La elevación de los valores de PCR puede ser útil a la hora de evaluar la gravedad de la infección; todos estos casos presentaban valores de PCR elevados. El recuento de leucocitos fue, en la mayoría de los casos, mayor a 15.000 céls $/ \mathrm{mm}^{3}$. Ningún paciente presentó leucopenia.

Las EI por $S$. pyogenes constituyen un importante problema de salud pública, dada la gravedad de estas entidades, con alta morbi-mortalidad. Requieren tratamientos médico-quirúrgicos, con estadías en unidades de cuidado intensivo e intermedio por períodos prolongados. En $40,6 \%$ de los casos analizados en esta serie fue necesaria la internación en UTI y en $60,6 \%$ hubo procedimientos quirúrgicos con fines terapéuticos. La mortalidad reportada en niños es de 5 a $20 \%{ }^{22}$. En esta serie de casos hubo un solo fallecimiento. Tal vez la baja mortalidad en esta serie se podría deber en parte a la ausencia de niños con varicela y al reducido número de casos con fascitis necrosante. Esta última constituye la forma de presentación más devastadora de esta enfermedad: en casi la mitad de los casos asocia SST y fallo multiorgánico, con una mortalidad entre 30 y $70 \%{ }^{2,7,19}$. Henriet y cols., analizaron 28 casos de niños con EI admitidos en un hospital pediátrico de Francia entre los años 2000 y 2007, obteniendo resultados similares a esta serie de casos; la infección osteo-articular fue la presentación clínica predominante $(15 / 28)$ en la serie francesa, al igual que lo observado en estos pacientes (15/32). También se registró un solo fallecimiento ${ }^{21}$.

Los tipos emm1, 3, 4 y 12 predominan en Estados Unidos de América y Europa ${ }^{21,23,24}$. Estos tipos emm, particularmente el 1 y 3 se han asociado a infecciones invasoras $^{25,26}$. En la serie presentada, las cepas con estos tipos emm tenían el gen que codifica para SpeA, exotoxina que actúa como superantígeno y es responsable de varias manifestaciones clínicas de la escarlatina y del síndrome de shock tóxico estreptocóccico. Estas cepas fueron aisladas de pacientes con cuadros clínicos graves de SST.

Estudios realizados previamente en el DLSP sobre cepas invasoras aisladas de niños reflejaron la misma distribución de tipos emm, perfil de toxinas y pulsotipos ${ }^{15}$. Cabe destacar que 3 de los 4 tipos emm (emm 1, 3 y 12) se encuentran en la formulación de una vacuna multivalente que aún no está disponible comercialmente ${ }^{27}$. Mantener la vigilancia epidemiológica de estas infecciones para conocer y divulgar la distribución nacional de tipos emm permitirá la toma de decisiones entre las diferentes fórmulas vacunales y servirá de base para la evaluación de la eficacia de la vacuna.

El conocimiento de las diversas presentaciones clínicas y su relación con las características moleculares son un desafío a futuro.

\section{Resumen}

Introducción: Streptococcus pyogenes, produce diversas manifestaciones clínicas. La enfermedad invasora (EI) se define por el aislamiento del microorganismo en sitios estériles. Objetivo: Analizar características clínicoepidemiológicas y moleculares de EI por $S$. pyogenes en niños hospitalizados en el HP-CHPR (1/1/05-31/1/13). Materiales y Métodos: Estudio descriptivo, retrospectivo, de casos con aislamiento de $S$. pyogenes de sitios estériles. Se analizaron variables epidemiológicas, características de la enfermedad, laboratorio, tratamientos, días de hospitalización y evolución. Se estudió la presencia de cuatro genes que codifican factores de virulencia y perfiles cromosómicos resueltos por electroforesis en campos pulsados. Resultados: Se detectó un total de 42 casos (tasa 4,6 cada 10.000 admisiones), se analizaron todas las variables en 32 . Mediana de edad 44,7 meses $(14<2$ años). En cinco se identificó puerta de entrada. Formas de presentación: infección osteo-articular (n: 15), shock (n: 6), infección de piel y tejidos blandos (n: 5), neumonía (n: 3) y bacteriemia (n: 3). Veinte requirieron procedimientos quirúrgicos y 13 de cuidado intensivo. La media de estadía hospitalaria fue de 17 días. Hubo un fallecimiento. Se realizó estudios moleculares en cinco cepas, identificándose cuatro perfiles de toxinas y pulsotipos diferentes. Discusión: La incidencia de EI en nuestra institución coincide con otras series en la región. Profundizar el conocimiento de la presentación clínica y su relación con las características moleculares es un desafío. 


\section{Referencias bibliográficas}

1.- Gerber M. Estreptococo grupo A. Kliegman B, Jenson S. Nelson Tratado de Pediatría, 19a ed. Barcelona: Elsevier; 2013, p. 955-65.

2.- Bisno A, Stevens D. Chapter N: Streptococcus pyogenes. Mandell, Douglas \& Bennett's Enfermedades Infecciosas, Principios y Prácticas, Mandell G L, Bennett J E, Dolin R, eds. 6ta ed. Madrid: Elsevier; 2006, p. 2362-76.

3.- Beall B, Facklam R. Sequencing emm-specific PCR products for routine and accurate typing of group A streptococci. J Clin Microbiol 1996; 34 : 953-8.

4.- Martin J, Green M \& M. Group A Streptococcus. Semin Pediatr Infect Dis 2006; 17: 140-8.

5.- Cunningham M. Pathogenesis of group $A$ streptococcal infections. Clin Microbiol Rev 2000; 13: 470-511.

6.- Picón T, Galazka J, Quian J, Bariani D, Rubio A, Lorenzo J. Infecciones pulmonares graves por estreptococo beta hemolítico del grupo A. Arch Pediatr Urug 1994; 65: 39-41.

7.- $\quad$ Prego J, Sehabiague G, de Leonardis D. Complicaciones graves de la varicela. Arch Pediatr Urug 1997; 68: 19-26.

8.- Prego J, Sehabiague G, de Leonardis D, Gutiérrez C. Varicela complicada con fascitis necrotizante. Importancia de un diagnóstico oportuno. Arch Pediatr Urug 2001; 72: 85-8.

9.- Isenberg H. Clinical Microbiology Procedures Handbook. Washington: American Society of Microbiology, 1994.

10.- The Working Group on Severe Streptococcal Infections. Defining the group A streptococcal toxic shock syndrome. Rationale and consensus definition. JAMA 1993; 269: 390-1.

11.- Kuppermann N. Bacteriemia oculta en niños febriles de corta edad. Pediatr Clin North Am 1999; 6: 1143-73.

12.- Pírez M C, Montano A, Rubio I, et al.
Neumonía. A Pírez M C, Montano A y Rubio. Atención Pediátrica. Normas nacionales de diagnóstico, tratamiento y prevención. $7^{\mathrm{a}} \mathrm{ed}$. Montevideo: Oficina del Libro FEFMUR 2008; 167-75.

13.- Tenover F C, Arbeit R D, Goering R V, Mickelsen P A, Murray B E, Persing D $\mathrm{H}$, et al. Interpreting chromosomal DNA restriction patterns produced by pulsed-field gel electrophoresis: criteria for bacterial strain typing. J Clin Microbiol 1995; 33 (9): 2233-9.

14.- Luca Harari B, Ekelund K. Clinical and epidemiological aspects of invasive Streptococcus pyogenes infections in Denmark during 2003 and 2004. J Clin Microbiol 2008; 46: $79-86$.

15.- García Gabarrot, G (2012). Identificación de linajes patogénicos en poblaciones naturales de Streptococcus pyogenes. Programa de Desarrollo de las Ciencias Básicas-PEDECIBA. Departamento de Laboratorios de Salud Pública, Montevideo, Uruguay. http://www.bib.fcien.edu. uy/files/etd/resumen/uy24-16150R.pdf

16.- Paganini H, Luppino V, Hernández C, Seú S, Debbag R. Infecciones invasivas por Streptococcus $\beta$-hemolítico del grupo A. Arch Argent Pediatr 2001; 99: 9-13.

17.- Alarcón C, Ordenes M, Denegri M, Zúñiga J. Infecciones invasoras por Streptococcus $\beta$ hemolítico Grupo A. Rev Chil Pediatr 2006; 77: 487-91.

18.- Bisno A L, Stevens D L. Streptococcus pyogenes. Mandell G L, Bennett J E and Dolin R (eds). Mandell, Douglas \& Bennett's Principles and Practice of Infectious Diseases New York: Churchill Livingstone 2000; 2101 17

19.- Kaplan E \& Gerber M. Group A, Group C and Group $\mathrm{G}$ beta-hemolytic streptococcal infections. Feigin, Cherry, Demmler-Harrison, Kaplan. Textbook of Pediatric Infectious Diseases. 6ta edición. Philadelphia: Elsevier 2009; 1225-39.

20.- Quian J. Actualizaciones del Certificado
Esquema de Vacunación: Perspectivas a futuro en vacunación en la niñez. Arch Pediatr Urug 2010; 81: 34-45.

21.- Henriet S, Kaguelidou F, Bidet P, Lorrot M, De Lauzanne A, Dauger S, et al. Invasive group A streptococcal infection in children: clinical manifestations and molecular characterization in a French pediatric tertiary care center. Eur $\mathbf{J}$ Clin Microbiol Infect Dis 2010; 29: 341-6.

22.- O'Loughlin R E, Roberson A, Cieslak P R, Lynfield R, Gershman K, Craig B, et al. The epidemiology of invasive group A streptococcal infection and potential vaccine implications: United States, 2000-2004. Clin Infect Dis 2005; 45: 853-62.

23.- O’Brien K L, Beall B, Barrett N L, Cieslak P R, Reingold A, Farley M M, et al. Epidemiology of invasive group A Streptococcus disease in the United States, 1995-1999. Clin Infect Dis 2002; 35: 268-76.

24.- Luca-Harari B, Darenberg J, Neal S, Siljander T, Strakova L, Tanna A, et al. Clinical and microbiological characteristics of severe Streptococcus pyogenes disease in Europe. J Clin Microbiol 2009; 47: 1155-65.

25.- Creti R, Gherardi G, Imperi M, von Hunolstein C, Baldassarri L, Pataracchia M, et al. Association of group A streptococcal emm types with virulence traits and macrolide-resistance genes is independent of the source of isolation. J Med Microbiol 2005; 54: 913-7.

26.- Montes M, Ardanuy C, Tamayo E, Domenech A, Linares J, Pérez-Trallero E, et al. Epidemiological and molecular analysis of Streptococcus pyogenes isolates causing invasive disease in Spain (1998-2009): comparison with non-invasive isolates. Eur J Clin Microbiol Infect Dis 2011; 30: 1295-302.

27.- $\mathrm{Hu}$ M C, Walls M A, Stroop S D, Reddish M A, Beall B, Dale J B, et al. Immunogenicity of a 26-valent group A streptococcal vaccine. Infect Immun 2002; 70 : 2171-7. 